\title{
Computation of Partially Invariant Solutions for the Einstein Walker Manifolds' Identifying Equations
}

\author{
Mehdi Nadjafikhaha,*, Mehdi Jafari ${ }^{\mathrm{b}}$ \\ ${ }^{a}$ School of Mathematics, Iran University of Science and Technology, Narmak, Tehran 1684613114, Iran \\ ${ }^{b}$ Department of Complementary Education, Payame Noor University, PO BOX 19395-3697, Tehran, Iran
}

\begin{abstract}
In this paper, partially invariant solutions (PISs) method is applied in order to obtain new four-dimensional Einstein Walker manifolds. This method is based on subgroup classification for the symmetry group of partial differential equations (PDEs) and can be regarded as the generalization of the similarity reduction method. For this purpose, those cases of PISs which have the defect structure $\delta=1$ and are resulted from two-dimensional subalgebras are considered in the present paper. Also it is shown that the obtained PISs are distinct from the invariant solutions that obtained by similarity reduction method.
\end{abstract}

Keywords: Einstein Walker manifolds, Lie symmetry group, Optimal system of Lie subalgebras, Partially invariant solutions (PISs)

2000 MSC: 70G65, 34C14, 53C50

\section{Introduction}

The idea of analyzing the differential equations by applying the transformation groups implied a new theory: the symmetry group theory, also called Lie group analysis. This method was originated at the end of nineteenth century from the pioneering work of Sophus Lie [1]. Since that time, several books have been dedicated to this concept and its generalizations [2-5]. Classification of the group invariant solutions and reduction of the original system can be regarded as significant applications of the Lie group method in the theory of differential equations. The fact that symmetry reductions for many PDEs can not be obtained via the classical symmetry method, motivated the creation of several generalizations of the classical Lie group method for symmetry reductions. Consequently, several alternative reduction methods have been proposed, going beyond Lie's classical procedure and providing further solutions. Partially invariant solutions (PISs) method is one of these procedures. This algorithmic method is a powerful tool for the reduction of PDEs and is based on classifying the subgroups of the symmetry group. The notion of PISs was first developed by Ovsiannikov [5] and can be considered as the extension of invariant solutions. The algorithm of constructing PISs is similar to that of invariant solutions. Indeed, obtaining the invariant solutions by applying the PISs method is easier than similarity reduction method whenever we deal with low-dimensional groups. One significant concept which appears while constructing PISs is the defect structure. This quantity is determined by the dimension of orbits and is denoted by $\delta$.

In this paper, the PISs method will be applied in order to construct some new classes of four-dimensional Einstein Walker manifolds. Those Manifolds which admit null parallel distributions are called Walker manifolds. A Walker manifold is called Einstein Walker manifold if its Ricci tensor is a scaler multiple of the metric at each point. Four-dimensional Einstein Walker manifolds form the underling structure of many geometric and physical models such as; hh-space in general relativity, pp-wave model and other areas [6-12].

*Corresponding author. Tel.:+98 21 73225426; Fax: +98 2173228426

Email addresses: m_nadjafikhah@iust.ac.ir (Mehdi Nadjafikhah), m.jafari@phd.pnu.ac.ir (Mehdi Jafari) 
The general form of the metric tensor of four-dimensional walker manifolds is expressed as follows [13]:

$$
\begin{aligned}
g_{a, b, c}:= & 2(d x \circ d y+d t \circ d z)+a(x, t, y, z) d y \circ d y \\
& +b(x, t, y, z) d z \circ d z+2 c(x, t, y, z) d y \circ d z,
\end{aligned}
$$

where $a, b$ and $c$ are smooth functions with respect to $(x, t, y, z)$. Let $M_{a, b, c}:=\left(\mathcal{O}, g_{a, b, c}\right)$, where $\mathcal{O}$ be an open subset of $\mathbb{R}^{4}$. We can see that $M_{a, b, c}$ is Einstein if and only if the functions $a, b$ and $c$ verify the following system of PDEs ([13], page 81).

$$
\begin{aligned}
& a_{11}-b_{22}=0, \quad b_{12}+c_{11}=0, \quad a_{12}+c_{22}=0, \\
& a_{1} c_{2}+a_{2} b_{2}-a_{2} c_{1}-c_{2}^{2}+2 c a_{12}+b a_{22}-2 a_{24}-a c_{12}+2 c_{23}=0, \\
& a_{2} b_{1}-c_{1} c_{2}+c a_{11}-a_{14}-b_{23}-a c_{11}-c c_{12}+c_{13}-b c_{22}+c_{24}=0, \\
& a_{1} b_{1}-b_{1} c_{2}+b_{2} c_{1}-c_{1}^{2}+a b_{11}+2 c b_{12}-2 b_{13}-b c_{12}+2 c_{14}=0,
\end{aligned}
$$

where the index 1,2, 3 and 4 for functions $a, b$ and $c$ represent the derivatives of these functions with respect to $x, t, y$ and $z$, respectively. The system (2) is hard to handle, so we consider a spacial case in this paper; where $a, b$ and $c$ only depend on $x$ and $t$. Therefore the following system must be solved.

$$
\begin{aligned}
& a_{11}-b_{22}=0, \quad b_{12}+c_{11}=0, \quad a_{12}+c_{22}=0 \\
& a_{1} c_{2}+a_{2} b_{2}-a_{2} c_{1}-c_{2}^{2}+2 c a_{12}+b a_{22}-a c_{12}=0 \\
& a_{2} b_{1}-c_{1} c_{2}+c a_{11}-a c_{11}-c c_{12}-b c_{22}=0 \\
& a_{1} b_{1}-b_{1} c_{2}+b_{2} c_{1}-c_{1}^{2}+a b_{11}+2 c b_{12}-b c_{12}=0
\end{aligned}
$$

In system (3), $x$ and $t$ are independent and $a, b$ and $c$ are dependent variables. It is worthwhile to say that some other special cases have been considered in many references which yield some results about the structures admitted by these manifolds [14-16].

In [17], we have comprehensively analyzed the problem of symmetries of the system (3). By applying the basic Lie symmetry method, we have obtained the classical Lie point symmetry operators of the system (3) and proved the following result (refer to [17] for more details):

Corollary 1.1. The Lie group of point symmetries of the PDE system (3) has a seven-dimensional Lie subalgebra generated by the following vector fields:

$$
\begin{array}{lll}
X_{1}=\partial_{x}, & X_{2}=\partial_{t}, & X_{3}=x \partial_{x}-2 b \partial_{b}-c \partial_{c}, \\
X_{4}=x \partial_{t}+2 c \partial_{b}+a \partial_{c}, & X_{5}=t \partial_{x}+2 c \partial_{a}+b \partial_{c}, & X_{6}=t \partial_{t}+2 b \partial_{b}+c \partial_{c} \\
X_{7}=a \partial_{a}+b \partial_{b}+c \partial_{c}, & &
\end{array}
$$

$\left(\partial_{x} \equiv \frac{\partial}{\partial x}, \ldots\right)$.

Mainly, we have constructed an optimal system of one-dimensional subalgebras in [17] which provides the preliminary classification of group invariant solutions for the system (3). Also, we have obtained the corresponding invariant solutions of this system via the method of similarity reduction.

It is worth considering that some of the partially invariant solutions are not invariant with respect to the subalgebras of lower dimensions. They are known as non-reducible PISs. By determining non-reducible PISs for the system (3), we can obtain some new four-dimensional Einstein Walker manifolds.

This paper is organized as follows: In section 2, we recall the general procedure of determining PISs. In section 3, we classify the two-dimensional subalgebras of the symmetry Lie algebra and construct an optimal system. Section 4 is devoted to the computation of some of the PISs associated to the system (3). In section 5 , the relation between PISs and invariant solutions is investigated and the condition for obtaining the non-reducible PISs is stated. Meanwhile, some non-reducible PISs for the system (3) are presented. Some concluding remarks are declared at the end of the paper.

\section{Partially invariant solutions method}

In this section, we recall the general procedure for determining PISs for an arbitrary system of PDEs. First, we present a brief review of the concept of PISs [18, 5]. Consider a system of PDEs of $n$th order with 
$p$ independent variables $\left(x=\left(x^{i}\right) \in X, i=1, \ldots, p\right)$ and $q$ dependent variables $\left(u=\left(u^{j}\right) \in U, j=1, \ldots, q\right)$ such as:

$$
\Delta=\Delta_{\mu}\left(x, u^{(n)}\right)=0, \quad \mu=1, \ldots, r .
$$

Let $G$ be a local symmetry group of the above system which acts on the total space $X \times U$ with $r$-dimensional orbits. If $u=f(x)$ is a solution of the system (5) with graph $\Gamma_{f}$, then the orbit space of $\Gamma_{f}$ can be defined as follows:

$$
G \Gamma_{f}=\left\{g \cdot(x, u) \mid(x, u) \in \Gamma_{f}, g \in G\right\}
$$

which is the union of the orbits of the $\Gamma_{f}$-elements.

The defect structure of the solution $u=f(x)$ with respect to the group $G$ is computed by the matrix of generators' characteristics and is defined as:

$$
\delta=\operatorname{dim}\left(G \Gamma_{f}\right)-\operatorname{dim}\left(\Gamma_{f}\right)=\operatorname{dim}\left(G \Gamma_{f}\right)-p .
$$

Also, we have $0 \leq \delta \leq \min \{r, q\}$ ([5], p 276-277).

If $\delta=0$, then $u=f(x)$ is an invariant solution and if $0<\delta<\min \{r, q\}$, then $u=f(x)$ is a partially invariant solution.

In order to calculate the PISs, first of all, it is necessary to classify the symmetry group into conjugacy classes. For obtaining the PISs with the defect structure $\delta$, those subgroups $H \subset G$ which have the property that if the dimension of the orbits of $H$ on the space $X \times U$ is $r$, then the dimension of the orbits is $r-\delta$ on the space $X$, must be selected [19]. Let $H$ be a subgroup with this property that mentioned above and $\mathfrak{h}$ be its Lie algebra with infinitesimal generators $\left\{v_{1}, \ldots, v_{s}\right\}$. Hence, we can obtain a complete set of functionally independent invariants of the form

$$
\left\{\xi_{i}(x), I_{j}(x, u)\right\}
$$

where $i=1, \ldots, p+\delta-s$ and $j=1, \ldots, q-\delta$.

Then we have

$$
\operatorname{rank}\left(\frac{\partial I_{j}(x, u)}{\partial u}\right)=q-\delta=q^{\prime} .
$$

If $u=f(x)$ is a function, then the manifold $H \Gamma_{f}$ can be expressed in terms of the invariants (8). So, we have:

$$
I_{j}(x, u)=f_{j}\left(\xi_{i}(x)\right)
$$

where the functions $f_{j}$ are arbitrary. Now, by applying the implicit function theorem, we conclude that

$$
u^{i_{\alpha}}=U^{i_{\alpha}}\left(x, u^{j \beta}, f_{j}\left(\xi_{i}(x)\right)\right)
$$

where $\alpha=1, \ldots, q^{\prime}$ and $\beta=1, \ldots, \delta$. The remaining dependent variables only depend on the original independent variables:

$$
u^{j_{\beta}}=U^{j_{\beta}}\left(x_{1}, \ldots, x_{p}\right), \quad \beta=1, \ldots, \delta .
$$

Now, the derivatives of the functions $u^{1}, \ldots, u^{q}$ with respect to the new variables which are obtained from equations (11) and (12) must be calculated. Hence, by substituting these quantities into the original system, a new system is obtained, involving the $q^{\prime}$ functions $f_{j}$ and the invariants $\xi_{i}$. The resulted equations are not generally consistent, so that the compatibility conditions must be computed. Consequently, a system of PDEs is deduced from these constraints which is denoted by $\Delta / H$. On the other hand, a system of PDEs is resulted from (12) denoted by $\Delta^{\prime}$. Now, the system $\Delta / H$ must be solved first. Then corresponding to each of the solution of this system, the system $\Delta^{\prime}$ will be solved. Finally, the partially invariant solutions are obtained by substituting the resulted solutions into equations (11) and (12). For more details about the methods of determining the PISs refer to [20, 5]. 


\section{Classification of Subalgebras for the system (3)}

In this section, we want to classify the subgroups of symmetry group of the system (3), into conjugacy classes. Searching for the invariant solutions can be regarded as the main motivation of computing the symmetries of a differential equation. As it is well known, the problem of classifying invariant solutions is equivalent to the problem of classifying the subgroups of the full symmetry group under conjugation. Let $H$ and $\tilde{H}$ be two connected, $s$-dimensional Lie subgroups of the Lie group $G$ with corresponding Lie subalgebras $\mathfrak{h}$ and $\tilde{\mathfrak{h}}$ of the Lie algebra $\mathfrak{g}$ of $G$. Let $g \in G$, then $\tilde{H}=g H g^{-1}$ are conjugate subgroups if and only if $\tilde{\mathfrak{h}}=\operatorname{Ad}(g) \cdot \mathfrak{h}$ are conjugate subalgebras, where $\operatorname{Ad}(g)$ is adjoint represen Hence, the problem of determining an optimal system of subgroups is equivalent to that of obtaining an optimal system of subalgebras, and so we focus on it [4, [5]. The latter problem, tends to obtain a list of conjugacy inequivalent subalgebras with the property that any other subalgebra is equivalent to only a unique member of the list under some element of the adjoint representation for some element of the investigated Lie group.

\subsection{Optimal system of one-dimensional subalgebras for the system (3)}

Indeed, for one-dimensional subalgebras, the classification problem is necessarily the same as the problem of classifying the orbits of the adjoint representation. Thus, an optimal set of subalgebras is constructed if we select just one representative from each family of equivalent subalgebras. Consequently, the associated set of invariant solutions is then the minimal list from which we can obtain all other invariant solutions of one-dimensional subalgebras simply via transformations.

In [17], we have presented a comprehensive analysis of this problem and have constructed an optimal system of one-dimensional subalgebras for the system (3) as follows :

Theorem 3.1. An optimal system of one-dimensional Lie subalgebras of the system (3) is provided by the following generators:
1) $\mathbf{X}^{1}=X_{7}$,
8) $\mathbf{X}^{8}=X_{4}+a X_{5}+b X_{6}+c X_{7}$,
2) $\mathbf{X}^{2}=X_{1}+a X_{7}$,
9) $\mathbf{X}^{9}=\varepsilon X_{1}+X_{4}+a X_{5}+b X_{6}+c X_{7}$,
3) $\mathbf{X}^{3}=X_{2}+a X_{7}$,
10) $\mathbf{X}^{10}=X_{3}+a X_{5}+b X_{6}+c X_{7}$,
4) $\mathbf{X}^{4}=X_{6}+a X_{7}$
11) $\mathbf{X}^{11}=\varepsilon X_{2}+X_{3}+a X_{5}+b X_{6}+c X_{7}$,
5) $\mathbf{X}^{5}=\varepsilon X_{1}+X_{6}+a X_{7}$
12) $\mathbf{X}^{12}=X_{3}+\varepsilon X_{4}+a X_{5}+b X_{6}+c X_{7}$,
6) $\mathbf{X}^{6}=X_{5}+a X_{6}+b X_{7}$,
13) $\mathbf{X}^{13}=\varepsilon X_{2}+X_{3}+\varepsilon^{\prime} X_{4}+a X_{5}+b X_{6}+c X_{7}$.
7) $\mathbf{X}^{7}=\varepsilon X_{2}+X_{5}+a X_{6}+b X_{7}$,

where $\varepsilon$ and $\varepsilon^{\prime}$ are \pm 1 and $a, b, c \in \mathbb{R}$ are arbitrary numbers [17].

\subsection{Optimal system of two-dimensional subalgebras for the system (3)}

In this paper, we need to classify the two-dimensional subalgebras. Because, we want to calculate those PISs which have the defect structure $\delta=1$ and the reduced system $\Delta / H$ is a system of ordinary differential equations. Since $p=2, \delta=1$ and $p+\delta-s=1$, then we have $s=2$. So, we should consider the twodimensional subgroups.

Consequently, constructing the two-dimensional optimal system, i.e., classification of the two-dimensional subalgebras of $\mathfrak{g}$ is our next step. This process is performed by choosing one of the vector fields as stated in theorem (3.1). Let us consider $\mathbf{X}^{1}$ (or $\mathbf{X}^{i}, i=2, \cdots, 13$ ). Corresponding to it, an optional vector field $Y=b_{1} X_{1}+\cdots+b_{7} X_{7}$ is selected, so we must have

$$
\left[\mathbf{X}^{1}, Y\right]=\lambda \mathbf{X}^{1}+\mu Y .
$$

Equation (14) leads us to the system

$$
C_{j k}^{i} \alpha_{j} a_{k}=\lambda a_{i}+\mu \alpha_{i} \quad(i=1, \cdots, 7) .
$$


where the constant coefficients $C_{j k}^{i}$ are the structure constants. The solutions of the system (15), give one of the two-dimensional generators and the second generator is $\mathbf{X}^{1}$ (or $\mathbf{X}^{i}, i=2, \cdots, 13$ ) if selected.

After the construction of all two-dimensional subalgebras, for every vector fields of theorem (3.1), they need to be simplified by the action of adjoint transformations in the manner analogous to the way of onedimensional optimal system. Hence, we can state the following theorem:

Theorem 3.2. Any two-dimensional subalgebras of (4) is conjugate to precisely one of the following subalgebras:

$$
\begin{aligned}
& \mathcal{A}_{1}^{1}:\left\langle X_{1}, X_{3}+\alpha X_{6}+\beta X_{7}\right\rangle, \\
& \mathcal{A}_{1}^{4}:\left\langle X_{1}, X_{3}+\epsilon X_{5}+X_{6}+\alpha X_{7}\right\rangle \text {, } \\
& \mathcal{A}_{1}^{2}:\left\langle X_{1}, X_{2}+\alpha X_{5}+\beta X_{7}\right\rangle, \\
& \mathcal{A}_{1}^{3}:\left\langle X_{1}, X_{5}+\alpha X_{7}\right\rangle, \\
& \mathcal{A}_{1}^{7}:\left\langle X_{1}, X_{7}\right\rangle \text {, } \\
& \mathcal{A}_{2}^{1}:\left\langle X_{2}, X_{3}+\alpha X_{6}+\beta X_{7}\right\rangle, \\
& \mathcal{A}_{2}^{4}:\left\langle X_{2}, X_{3}+\epsilon X_{4}+X_{6}+\alpha X_{7}\right\rangle \text {, } \\
& \mathcal{A}_{2}^{7}:\left\langle X_{2}, X_{7}\right\rangle \text {, } \\
& \mathcal{A}_{3}^{1}:\left\langle X_{6}, X_{3}+\alpha X_{7}\right\rangle, \\
& \mathcal{A}_{3}^{4}:\left\langle X_{6}, X_{1}+\alpha X_{7}\right\rangle \text {, } \\
& \mathcal{A}_{4}^{1}:\left\langle\varepsilon X_{1}+X_{6}, X_{2}\right\rangle \text {, } \\
& \mathcal{A}_{5}^{1}:\left\langle X_{5}, X_{3}+\alpha X_{6}+\beta X_{7}\right\rangle, \\
& \mathcal{A}_{5}^{4}:\left\langle X_{5}, X_{7}\right\rangle \text {, } \\
& \mathcal{A}_{6}^{1}:\left\langle\varepsilon X_{2}+X_{5}, X_{3}+\frac{1}{2} X_{6}+\alpha X_{7}\right\rangle, \\
& \mathcal{A}_{7}^{1}:\left\langle X_{4}, X_{3}+\alpha X_{6}+\beta X_{7}\right\rangle, \\
& \mathcal{A}_{7}^{4}:\left\langle X_{4}, X_{7}\right\rangle \text {, } \\
& \mathcal{A}_{8}^{1}:\left\langle\varepsilon X_{1}+X_{4}, X_{3}+2 X_{6}+\alpha X_{7}\right\rangle, \\
& \mathcal{A}_{9}^{1}:\left\langle X_{3}, X_{2}+\alpha X_{7}\right\rangle, \\
& \mathcal{A}_{9}^{4}:\left\langle X_{3}, X_{6}+\alpha X_{7}\right\rangle \text {, } \\
& \mathcal{A}_{10}^{1}:\left\langle\varepsilon X_{2}+X_{3}, X_{1}\right\rangle \\
& \mathcal{A}_{10}^{4}:\left\langle X_{2}, X_{3}+\alpha X_{7}\right\rangle \text {, } \\
& \mathcal{A}_{11}^{1}:\left\langle X_{3}+\varepsilon X_{4}, \varepsilon X_{5}+X_{6}-2 X_{7}\right\rangle, \\
& \mathcal{A}_{11}^{4}:\left\langle X_{3}+\varepsilon X_{4}, X_{3}+X_{6}+\alpha X_{7}\right\rangle \text {, } \\
& \mathcal{A}_{2}^{2}:\left\langle X_{2}, X_{1}+\epsilon X_{4}+\beta X_{7}\right\rangle, \\
& \mathcal{A}_{2}^{5}:\left\langle X_{2}, X_{1}+X_{6}+\alpha X_{7}\right\rangle \text {, } \\
& \mathcal{A}_{2}^{3}:\left\langle X_{2}, X_{4}+\alpha X_{7}\right\rangle, \\
& \mathcal{A}_{2}^{6}:\left\langle X_{2}, X_{6}+\alpha X_{7}\right\rangle, \\
& \mathcal{A}_{3}^{2}:\left\langle X_{6}, X_{4}\right\rangle, \\
& \mathcal{A}_{3}^{5}:\left\langle X_{6}, X_{2}\right\rangle \text {, } \\
& \mathcal{A}_{3}^{3}:\left\langle X_{6}, X_{5}\right\rangle, \\
& \mathcal{A}_{3}^{6}:\left\langle X_{6}, X_{7}\right\rangle \text {, } \\
& \mathcal{A}_{4}^{2}:\left\langle\varepsilon X_{1}+X_{6}, X_{5}\right\rangle, \\
& \mathcal{A}_{5}^{2}:\left\langle X_{5}, X_{1}+\alpha X_{6}+\beta X_{7}\right\rangle \text {, } \\
& \mathcal{A}_{4}^{3}:\left\langle\varepsilon X_{1}+X_{6}, X_{7}\right\rangle \text {, } \\
& \mathcal{A}_{5}^{3}:\left\langle X_{5}, X_{6}+\alpha X_{7}\right\rangle \text {, } \\
& \mathcal{A}_{6}^{2}:\left\langle\varepsilon X_{2}+X_{5}, X_{1}+\alpha X_{7}\right\rangle, \\
& \mathcal{A}_{6}^{3}:\left\langle\varepsilon X_{2}+X_{5}, X_{7}\right\rangle, \\
& \mathcal{A}_{7}^{2}:\left\langle X_{4}, X_{2}+\alpha X_{3}+\beta X_{7}\right\rangle, \\
& \mathcal{A}_{7}^{3}:\left\langle X_{4}, X_{6}+\alpha X_{7}\right\rangle, \\
& \mathcal{A}_{8}^{2}:\left\langle\varepsilon X_{1}+X_{4}, X_{2}+\alpha X_{7}\right\rangle, \\
& \mathcal{A}_{8}^{3}:\left\langle\varepsilon X_{1}+X_{4}, X_{7}\right\rangle, \\
& \mathcal{A}_{9}^{2}:\left\langle X_{3}, X_{5}\right\rangle, \\
& \mathcal{A}_{9}^{5}:\left\langle X_{3}, X_{7}\right\rangle \text {, } \\
& \mathcal{A}_{10}^{2}:\left\langle\varepsilon X_{2}+X_{3}, X_{4}\right\rangle \text {, } \\
& \mathcal{A}_{9}^{3}:\left\langle X_{3}, X_{1}\right\rangle \text {, } \\
& \mathcal{A}_{9}^{6}:\left\langle X_{3}, X_{4}\right\rangle \text {, } \\
& \mathcal{A}_{10}^{3}:\left\langle\varepsilon X_{2}+X_{3}, X_{7}\right\rangle \text {, } \\
& \mathcal{A}_{12}^{1}:\left\langle\varepsilon X_{2}+X_{3}+\varepsilon^{\prime} X_{4}, X_{1}+\varepsilon^{\prime} X_{2}\right\rangle, \quad \mathcal{A}_{12}^{2}:\left\langle\varepsilon X_{2}+X_{3}+\varepsilon^{\prime} X_{4}, X_{7}\right\rangle, \\
& \mathcal{A}_{11}^{3}:\left\langle X_{3}+\varepsilon X_{4}, X_{7}\right\rangle \\
& \mathcal{A}_{11}^{2}:\left\langle X_{3}+\varepsilon X_{4}, X_{2}+\alpha X_{7}\right\rangle, \\
& \mathcal{A}_{11}^{5}:\left\langle X_{3}+\varepsilon X_{4}, X_{1}+\varepsilon X_{2}\right\rangle \text {, }
\end{aligned}
$$

where $\alpha$ and $\beta$ are arbitrary constants, $\varepsilon$ and $\varepsilon^{\prime}$ are \pm 1 and $\epsilon$ is \pm 1 or 0 .

Proof. Each of the two-dimensional subalgebra has two generators. For classifying two-dimensional subalgebras, we must select one of the generators from the list of one-dimensional optimal system (13) and another generator must be taken optionally.

Suppose that $\mathfrak{h}=\operatorname{span}\{X, Y\}$ is a two-dimensional subalgebra of $\mathfrak{g}$ where $X$ is a one-dimensional subalgebra which is chosen from the list (13) and $Y$ is an optional vector defined by: $Y=b_{1} X_{1}+\cdots+b_{7} X_{7}$. Now, we must simplify $\mathfrak{h}$ as much as possible by imposing various adjoint transformations on it [4].

Each adjoint transformation is a linear map $F_{i}^{s}: \mathfrak{g} \rightarrow \mathfrak{g}$ defined by $X \mapsto \operatorname{Ad}\left(\exp \left(s X_{i}\right) . X\right)$, for $i=1, \cdots, 7$. 
Since this procedure is so lengthy, we only explain one of the cases in the following.

Case1 : If $X=X_{1}+a X_{7}$ then

$$
\begin{aligned}
\mathfrak{h}=\langle X, Y\rangle & =\left\langle X_{1}+a X_{7}, \sum_{i=1}^{7} b_{i} X_{i}\right\rangle \\
& =\left\langle X_{1}, b_{2} X_{2}+b_{3} X_{3}+b_{4} X_{4}+b_{5} X_{5}+b_{6} X_{6}+b_{7} X_{7}\right\rangle
\end{aligned}
$$

So, we have:

a) If $b_{2}=b_{3}=b_{4}=b_{5}=b_{6}=0$, then we have $\mathfrak{h}=\left\langle X_{1}, b_{7} X_{7}\right\rangle=\left\langle X_{1}, X_{7}\right\rangle$. Since $\left[X_{1}, X_{7}\right]=0=$ $0 X_{1}+0 X_{7}$, then $\mathfrak{h}$ is closed under the Lie bracket. So, $\mathfrak{h}$ is reduced to the case $\mathcal{A}_{1}^{7}$.

b) If $b_{2}=b_{3}=b_{4}=b_{5}=0$ and $b_{6} \neq 0$, then $\mathfrak{h}=\left\langle X_{1}, b_{6} X_{6}+b_{7} X_{7}\right\rangle=\left\langle X_{1}, X_{6}+\alpha X_{7}\right\rangle$. Since $\left[X_{1}, X_{6}+\alpha X_{7}\right]=0$, then $\mathfrak{h}$ is closed under the Lie bracket. So, $\mathfrak{h}$ is reduced to the case $\mathcal{A}_{1}^{6}$.

c) If $b_{2}=b_{3}=b_{4}=b_{6}=0$ and $b_{5} \neq 0$, then $\mathfrak{h}=\left\langle X_{1}, b_{5} X_{5}+b_{7} X_{7}\right\rangle=\left\langle X_{1}, X_{5}+\alpha X_{7}\right\rangle$. Since $\left[X_{1}, X_{5}+\alpha X_{7}\right]=0$, then $\mathfrak{h}$ is closed under the Lie bracket. So, $\mathfrak{h}$ is reduced to the case $\mathcal{A}_{1}^{3}$.

d) If $b_{2}=b_{3}=b_{4}=0$ and $b_{6} \neq 0$, then we can make the coefficient of $X_{5}$ vanish by $F_{5}^{s_{5}}$; By setting $s_{5}=\frac{1}{b_{6}}$. So, we have $\mathfrak{h}=\left\langle X_{1}, b_{6} X_{6}+b_{7} X_{7}\right\rangle$ which is reduced to the case $\mathcal{A}_{1}^{6}$ like (b).

e) If $b_{2}=b_{3}=0$ and $b_{4} \neq 0$, then $\mathfrak{h}=\left\langle X_{1}, b_{4} X_{4}+\cdots+b_{7} X_{7}\right\rangle$ and $\left[X_{1}, b_{4} X_{4}+\cdots+b_{7} X_{7}\right]=b_{4} X_{2} \neq$ $r X_{1}+s\left(b_{4} X_{4}+\cdots+b_{7} X_{7}\right)$ for any $r, s \in \mathbb{R}$. So, $\mathfrak{h}$ is not closed under the Lie bracket and we have not any two-dimensional subalgebras in this case.

f) If $b_{2}=b_{4}=0, b_{3} \neq 0$ and $b_{6} \neq 1$, then we can make the coefficient of $X_{5}$ vanish by $F_{5}^{s_{5}}$; By setting $s_{5}=\frac{b_{5}}{-1+b_{6}}$. Also, by scaling if necessary, we can assume that $b_{3}=1$. Then we have $\mathfrak{h}=$ $\left\langle X_{1}, X_{3}+b_{6} X_{6}+b_{7} X_{7}\right\rangle$ and $\left[X_{1}, X_{3}+b_{6} X_{6}+b_{7} X_{7}\right]=X_{1}$. So, $\mathfrak{h}$ is closed under the Lie bracket and the case $\mathcal{A}_{1}^{1}$ is concluded.

g) If $b_{2}=b_{4}=0, b_{3} \neq 0$ and $b_{6}=1$, then by scaling if necessary, we can assume that $b_{3}=1$. Also, the coefficient of $X_{5}$ can be vanished or be \pm 1 by $F_{6}^{s_{6}}$; By setting $s_{6}=-\ln \left|b_{5}\right|$. Then we have $\mathfrak{h}=\left\langle X_{1}, X_{3}+\varepsilon X_{5}+X_{6}+b_{7} X_{7}\right\rangle$ and $\left[X_{1}, X_{3}+\varepsilon X_{5}+X_{6}+b_{7} X_{7}\right]=X_{1}$. So, $\mathfrak{h}$ is closed under the Lie bracket and the case $\mathcal{A}_{1}^{4}$ is deduced.

h) If $b_{3}=b_{4}=b_{6}=0$ and $b_{2} \neq 0$, then by scaling if necessary, we can assume that $b_{2}=1$. Also, we have $\mathfrak{h}=\left\langle X_{1}, X_{2}+b_{5} X_{5}+b_{7} X_{7}\right\rangle$ and $\left[X_{1}, X_{2}+b_{5} X_{5}+b_{7} X_{7}\right]=0$. So, $\mathfrak{h}$ is closed under the Lie bracket and the case $\mathcal{A}_{1}^{2}$ is resulted.

i) If $b_{4}=b_{6}=0, b_{2} \neq 0$ and $b_{3} \neq 0$, then by scaling if necessary, we can assume that $b_{2}=1$. Also we can make the coefficient of $X_{5}$ vanish by $F_{5}^{s_{5}}$; By setting $s_{5}=-\frac{b_{5}}{b_{3}}$. Then we have $\mathfrak{h}=$ $\left\langle X_{1}, X_{2}+b_{3} X_{3}+b_{7} X_{7}\right\rangle$ and $\left[X_{1}, X_{2}+b_{3} X_{3}+b_{7} X_{7}\right]=b_{3} X_{1}$. So, $\mathfrak{h}$ is closed under the Lie bracket and the case $\mathcal{A}_{1}^{5}$ is resulted .

j) If $b_{4}=0, b_{6} \neq 0$ and $b_{3}=1$, then by scaling if necessary, we can assume that $b_{6}=1$. We can make the coefficient of $X_{2}$ vanish by $F_{2}^{s_{2}}$; By setting $s_{2}=-\frac{1}{b_{6}}$. Also, the coefficient of $X_{5}$ can be vanished or be \pm 1 by $F_{6}^{s_{6}}$; By setting $s_{6}=-\ln \left|\mathrm{b}_{5}\right|$. So, we have $\mathfrak{h}=\left\langle X_{1}, X_{3}+\varepsilon X_{5}+X_{6}+b_{7} X_{7}\right\rangle$ which is reduced to the case $\mathcal{A}_{1}^{4}$ similar to (g).

k) If $b_{4}=0, b_{6} \neq 0$ and $b_{3} \neq 1$, then we can make the coefficient of $X_{2}$ and $X_{5}$ vanish by $F_{2}^{s_{2}}$ and $F_{5}^{s_{5}}$; By setting $s_{2}=-\frac{1}{b_{6}}$ and $s_{5}=-\frac{b_{5}}{b_{3}-1}$, respectively. By scaling if necessary, we have $\mathfrak{h}=$ $\left\langle X_{1}, X_{3}+b_{6} X_{6}+b_{7} X_{7}\right\rangle$ that is reduced to the case $\mathcal{A}_{1}^{1}$ similar to (f).

In each case, $\mathfrak{h}$ can not be simplified any more by $F_{i}^{s_{i}}, i=1, \ldots, 7$. By a similar method, we can find the two-dimensional subalgebras for the other 11 cases. 


\section{Computation of the partially invariant solutions for the system (3)}

In this section, we will calculate the PISs of the system (3). For example, consider the Lie subalgebra $\mathcal{A}_{1}^{7}:\left\langle X_{1}, X_{7}\right\rangle$. The set of functionally independent invariants for $\mathcal{A}_{1}^{7}$ is a set of functions $I$ with the following property: $X_{1}(I)=X_{7}(I)=0$. By calculating these functions, a set of functionally independent invariants is obtained as follows:

$$
\left\{t, \frac{b}{a}, \frac{c}{a}\right\}
$$

So, we have:

$$
\operatorname{rank}\left(\frac{\partial\left(\frac{b}{a}, \frac{c}{a}\right)}{\partial(a, b, c)}\right)=\operatorname{rank}\left(\begin{array}{cc}
-b / a^{2} & -c / a^{2} \\
1 / a & 0 \\
0 & 1 / a
\end{array}\right)=2=q-\delta .
$$

Since $q=3$, then $\delta=1$. The equations corresponding to the relation (10) are

$$
\frac{b}{a}=f(t), \quad \frac{c}{a}=g(t),
$$

and the solutions corresponding to the equations (11) and (12) are expressed as follows:

$$
b=a f(t), \quad c=a g(t), \quad a=a(x, t) .
$$

Note that $a(x, t)$ is an arbitrary function.

Now, we can compute the derivatives of the functions $a, b$ and $c$ from relations (20):

$$
\begin{array}{llll}
b_{1}=a_{1} f, & b_{2}=a_{2} f+a f^{\prime}, & b_{11}=a_{11} f, & b_{22}=a_{22} f+2 a_{2} f^{\prime}+a f^{\prime \prime}, \\
c_{1}=a_{1} g, & c_{2}=a_{2} g+a g^{\prime}, & c_{11}=a_{11} g, & c_{22}=a_{22} g+2 a_{2} g^{\prime}+a g^{\prime \prime}, \\
b_{12}=a_{12} f+a_{1} f^{\prime}, & c_{12}=a_{12} g+a_{1} g^{\prime} . & &
\end{array}
$$

Hence, by substituting the above relations into the system (3), we obtain:

$$
\begin{aligned}
& a_{11}-a_{22} f-2 a_{2} f^{\prime}-a f^{\prime \prime}=0, a_{12} f+a_{1} f^{\prime}+a_{11} g=0, \\
& a_{12}+a_{22} g+2 a_{2} g^{\prime}+a g^{\prime \prime}=0, \quad a_{2}^{2} f+a_{2} a f^{\prime}-\left(a_{2} g+a g^{\prime}\right)^{2}+a a_{12} g+a a_{22} f=0, \\
& a_{1} a_{2} f-a_{1} a_{2} g^{2}-2 a a_{1} g g^{\prime}-a a_{12} g^{2}-a a_{22} f g-2 a a_{2} f g^{\prime}-a^{2} f g^{\prime \prime}=0, \\
& a_{1}^{2} f-2 a a_{1} f g^{\prime}-a_{1}^{2} g^{2}+a a_{11} f+3 a a_{1} g f^{\prime}+a a_{12} f g=0 .
\end{aligned}
$$

The consistency conditions, obtained from the system (22), conclude the following equations

$$
\begin{aligned}
& a^{2} f^{\prime \prime}+a a_{2} f^{\prime}+a^{2} g^{\prime 2}-a_{2}^{2} f+a_{2}^{2} g^{2}+2 a a_{2} g g^{\prime}=0, \quad a_{1}=0, \\
& a a_{22} f+a_{2}^{2} f-2 a a_{2} g g^{\prime}+a a_{2} f^{\prime}-a^{2} g^{\prime 2}-a_{2}^{2} g^{2}=0, \\
& a^{2} f g^{\prime \prime}-a a_{2} f^{\prime} g+a^{2} g g^{\prime 2}-a_{2}^{2} f g+a_{2}^{2} g^{3}+2 a a_{2} g^{\prime} g^{2}+2 a a_{2} f g^{\prime}=0,
\end{aligned}
$$

and these inequations

$$
f \neq 0, \quad g \neq 0, \quad a \neq 0, \quad f-g^{2} \neq 0 .
$$

Equations (23) form a system of ODEs. By solving this system, we obtain four types of solutions as follows:
1) $\left\{\begin{array}{l}f=c_{3} t+c_{4} \\ g=c_{2} \\ a=c_{1}\end{array}\right.$
2) $\left\{\begin{array}{l}f=\frac{c_{1} c_{3}^{2} t+c_{5}}{c_{1} t+c_{2}} \\ g=c_{3}+\frac{c_{1} c_{4}}{c_{1} t+c_{2}} \\ a=c_{1} t+c_{2}\end{array}\right.$
$\left\{\begin{aligned} f & =\frac{c_{5}\left(t+c_{2}\right)}{\ln \left(t+c_{2}\right)-c_{3} c_{1}} \\ g & =\frac{c_{4}}{\ln \left(t+c_{2}\right)-c_{3} c_{1}} \\ a & =-\frac{\ln \left(t+c_{2}\right)}{c_{1}}+c_{3}\end{aligned}\right.$
$\left\{\begin{array}{l}f=\frac{c_{6}^{2}\left(t+c_{2}\right)}{\left(c_{1} \ln \left(t+c_{2}\right)+c_{3} t+c_{4}\right) c_{3}} \\ g=\frac{c_{5}+c_{6} t}{c_{1} \ln \left(t+c_{2}+c_{3} t+c_{4}\right.} \\ a=c_{1} \ln \left(t+c_{2}\right)+c_{3} t+c_{4}\end{array}\right.$ 
Now, by applying the relations (20), the partially invariant solutions associated to the subalgebra $\mathcal{A}_{1}^{7}$ can be obtained for the system (3).
1) $\left\{\begin{array}{l}a=c_{1} \\ b=c_{1}\left(c_{3} t+c_{4}\right) \\ c=c_{1} c_{2}\end{array}\right.$
2) $\left\{\begin{array}{l}a=c_{1} t+c_{2} \\ b=c_{1} c_{3}^{2} t+c_{5} \\ c=c_{3}\left(c_{1} t+c_{2}\right)+c_{1} c_{4}\end{array}\right.$
3) $\left\{\begin{array}{l}a=\frac{-\ln \left(t+c_{2}\right)}{c_{1}}+c_{3} \\ b=c_{5}\left(t+c_{2}\right) \\ c=c_{4}\end{array}\right.$
4) $\left\{\begin{aligned} a & =c_{1} \ln \left(t+c_{2}\right)+c_{3} t+c_{4} \\ b & =\frac{c_{6}^{2}\left(t+c_{2}\right)}{c_{3}} \\ c & =c_{5}+c_{6} t\end{aligned}\right.$

These partially invariant solutions seem to be trivial. In a similar way, we can calculate nontrivial PISs by applying other subalgebras listed in theorem (3.2). Some other of these PISs are presented in Table 1.

\section{Non-reducible partially invariant solutions}

In this section, we will deal with those partially invariant solutions which are not invariant with respect to some of the subgroups of $G$. They are called non-reducible PISs and they are usually rare. For a reducible partially invariant solution $u=f(x)$, we can determine a subgroup $H^{\prime} \subset H$ which $u$ is a $H^{\prime}$-invariant solution and $\operatorname{dim}\left(H^{\prime} \Gamma_{f}\right) \geqslant \operatorname{dim}(H)-\delta=s-\delta([\underline{5}$, page 290). So, reducible PISs can be obtained via the method of similarity reduction from the reduced system involving $p-\operatorname{dim}\left(H^{\prime} \Gamma_{f}\right) \leqslant p+\delta-s$ independent variables, which is indeed easier than obtaining them by PISs method. For example, the PISs that we computed in the previous section are non-reducible PISs. Because, reducible PISs must be invariant with respect to the one-parameter subgroups of its Lie group. These subgroups have Lie subalgebras of $\mathcal{A}_{1}^{7}$ generated by an infinitesimal generator of the form $\alpha X_{1}+\beta X_{7}$ where $\alpha, \beta \in \mathbb{R}$. In [17], we obtained the invariant solutions of the system (3) with respect to this generator by applying the method of similarity reduction:
1) $\left\{\begin{array}{l}a=0 \\ b=0 \\ c=0\end{array}\right.$
2) $\left\{\begin{array}{l}a=0 \\ b=c_{1} e^{\frac{\beta}{\alpha} x} \\ c=0\end{array}\right.$
3) $\left\{\begin{array}{l}a=c_{2} e^{c_{1} t+\frac{\beta}{\alpha} x} \\ b=-\frac{c_{2} \beta}{c_{1} \alpha} e^{c_{1} t+\frac{\beta}{\alpha} x} \\ c=-\frac{c_{1} c_{2} \alpha}{\beta} e^{c_{1} t+\frac{\beta}{\alpha} x}\end{array}\right.$

Since, none of these solutions is similar to the PISs in (26), we conclude that PISs in (26) are non-reducible PISs. Several other non-reducible partially invariant solutions of the system (3) are listed in the following table.

Table 1: Non-reducible partially invariant solutions of (3).

\begin{tabular}{|c|c|c|c|}
\hline Subalgebras & Invariants & Dependent variables & PISs \\
\hline \multirow{3}{*}{$\left\langle X_{2}, X_{7}\right\rangle$} & \multirow{3}{*}{$x, \frac{b}{a}, \frac{c}{a}$} & $a=a(x, t)$ & $a=c_{1} x+c_{2}$ \\
\hline & & $b=a f(x)$ & $b=c_{1} c_{3}^{2} x+(*)$ \\
\hline & & $c=a g(x)$ & $c=c_{3}\left(c_{1} x+c_{2}\right)+c_{1} c_{4}$ \\
\hline$\left\langle X_{5}, X_{7}\right\rangle$ & $\begin{array}{l}\left\{t, \frac{c^{2}-b a}{b^{2}}\right. \\
\left.\frac{-c t+b x}{b}\right\}\end{array}$ & $\begin{array}{l}a=\frac{b(x-g(t))^{2}}{t^{2}}-b f(t) \\
b=b(x, t) \\
c=\frac{b}{t}(x-g(t))\end{array}$ & $\begin{array}{l}a=\frac{\left(c_{1}+c_{2} t^{3}\right)\left(x-c_{3}\right)^{2}}{t^{3}}-(* *) \\
b=\frac{c_{1}+c_{2} t^{3}}{t} \\
c=\frac{\left(c_{1}+c_{2} t^{3}\right)\left(x-c_{3}\right)}{t^{2}}\end{array}$ \\
\hline$\left\langle X_{2}, X_{6}+X_{7}\right\rangle$ & $\left\{x, \frac{b}{a^{3}}, \frac{c}{a^{2}}\right\}$ & $\begin{array}{l}a=a(x, t) \\
b=a^{3} f(x) \\
c=a^{2} g(x)\end{array}$ & $\begin{array}{l}a=\frac{4\left(t+c_{1}\right)}{\left(c_{2} x+c_{3}\right)^{2}} \\
b=\frac{4 c_{2}^{2}\left(t+c_{1}\right)^{3}}{\left(c_{2} x+c_{3}\right)^{4}} \\
c=\frac{4 c_{2}\left(t+c_{1}\right)^{2}}{\left(c_{2} x+c_{3}\right)^{3}}\end{array}$ \\
\hline$\left\langle X_{4}, X_{7}\right\rangle$ & $\begin{array}{l}\left\{x, \frac{-a t+c x}{x a},\right. \\
\left.\frac{a t^{2}-2 x t c+b x^{2}}{x^{2} a}\right\}\end{array}$ & $\begin{aligned} a & =a(x, t) \\
b & =a\left(g(x)+2 \frac{t}{x} f(x)+\frac{t^{2}}{x^{2}}\right) \\
c & =a\left(f(x)+\frac{t}{x}\right)\end{aligned}$ & $\begin{array}{l}a=\frac{c_{1}+c_{2} x^{3}}{x} \\
b=\frac{\left(c_{1}+c_{2} x^{3}\right)\left(t+c_{3}\right)^{2}}{x^{3}}+(* * *) \\
c=\frac{\left(c_{1}+c_{2} x^{3}\right)\left(t+c_{3}\right)}{x^{2}}\end{array}$ \\
\hline
\end{tabular}


where $c_{i}$ 's are arbitrary constants and

$$
\begin{aligned}
& *=\left(\frac{c_{5}}{c_{1}}-c_{3}^{2} c_{2}\right) \ln \left(c_{1} x+c_{2}\right)+c_{6}, \\
& * *=t\left(c_{4}+c_{5}\right)\left(\ln \left(t+\left(\frac{c_{1}}{c_{2}}\right)^{\left(\frac{1}{3}\right)}\right)^{2}-\ln \left(t^{2}-t\left(\frac{c_{1}}{c_{2}}\right)^{\left(\frac{1}{3}\right)}+\left(\frac{c_{1}}{c_{2}}\right)^{\left(\frac{2}{3}\right)}\right)+2 \sqrt{3} \tan ^{-1}\left(\frac{2 c_{2} t}{\sqrt{3} c_{1}}\left(\frac{c_{1}}{c_{2}}\right)^{\left(\frac{2}{3}\right)}-\frac{1}{\sqrt{3}}\right)\right), \\
& * * *=x\left(c_{4}+c_{5}\right)\left(\ln \left(x+\left(\frac{c_{1}}{c_{2}}\right)^{\left(\frac{1}{3}\right)}\right)^{2}-\ln \left(x^{2}-x\left(\frac{c_{1}}{c_{2}}\right)^{\left(\frac{1}{3}\right)}+\left(\frac{c_{1}}{c_{2}}\right)^{\left(\frac{2}{3}\right)}\right)+2 \sqrt{3} \tan ^{-1}\left(\frac{2 c_{2} x}{\sqrt{3} c_{1}}\left(\frac{c_{1}}{c_{2}}\right)^{\left(\frac{2}{3}\right)}-\frac{1}{\sqrt{3}}\right)\right) .
\end{aligned}
$$

\section{Conclusion}

Partially invariant solutions can be regarded as the natural extension of the invariant ones and the method of obtaining them is algorithmic. Some of the PISs are not invariant with respect to the lower dimensional subalgebras. They are called non-reducible PISs and can be applied in order to obtain some new solutions for an arbitrary system of PDEs. For example, in the present paper, we have obtained a non-reducible PISs for the system (3) as follows:

$$
a=\frac{4\left(t+c_{1}\right)}{\left(c_{2} x+c_{3}\right)^{2}}, \quad b=\frac{4 c_{2}^{2}\left(t+c_{1}\right)^{3}}{\left(c_{2} x+c_{3}\right)^{4}}, \quad c=\frac{4 c_{2}\left(t+c_{1}\right)^{2}}{\left(c_{2} x+c_{3}\right)^{3}}
$$

Consequently, according to relation (1), we could determine a general form for the metric associated to a set of four-dimensional Einstein Walker manifolds as follows:

$$
\begin{aligned}
g_{a, b, c}:= & 2(d x \circ d y+d t \circ d z)+\frac{4\left(t+c_{1}\right)}{\left(c_{2} x+c_{3}\right)^{2}} d y \circ d y \\
& +\frac{4 c_{2}^{2}\left(t+c_{1}\right)^{3}}{\left(c_{2} x+c_{3}\right)^{4}} d z \circ d z+\frac{8 c_{2}\left(t+c_{1}\right)^{2}}{\left(c_{2} x+c_{3}\right)^{3}} d y \circ d z .
\end{aligned}
$$

This set of solutions can not be obtained from the similarity reduction method. In a similar manner, we can deduce some other four-dimensional Einstein Walker manifolds from Table 1.

\section{Acknowledgements}

It is a pleasure to thank the anonymous referees for their constructive suggestions and helpful comments which have improved the presentation of the paper. The authors wish to express their sincere gratitude to Fatemeh Ahangari for her useful advise and suggestions.

\section{References}

[1] S. Lie, On integration of a class of linear partial differential equations by means of definite integrals, translation by N.H. Ibragimov, Arch Math. 6 (1881) 328-368.

[2] G. Bluman, A.F. Cheviakov, S. Anco, Application of symmetry methods to partial differential equations, Springer, New York, 2010

[3] A. Kushner, V. Lychagin, V. Rubstov, Contact geometry and nonlinear differential equations, Cambridge University Press, Cambridge, 2007.

[4] P.J. Olver, Applications of Lie Groups to Differential Equations, Springer, New York, 1986.

[5] L.V. Ovsiannikov, Group Analysis of Differential Equations, Academic Press. New York, 1982.

[6] C.P. Boyer, J. D. Finley III, and J. F. Plebański, Complex general relativity, $h$ and $h h$ spaces - a survey of one approach, Gen. Relativity Gravitation, 2 (1980) 241-281, Plenum, New York-London.

[7] J.D. Finley III, and J. F. Plebański, The intrinsic spinorial structure of hyperheavens, J. Math. Phys. 17 (1976), $2207-2214$.

[8] R. Abounasr, A. Belhaj, J. Rasmussen, and E.H. Saidi, Superstring theory on pp-waves with ADE geometries, J. Phys. A 39 (2006), 2797-2841.

[9] J. Kerimo, AdS pp-waves, J. High Energy Phys. (2005), 025, 18 pp.

[10] J. Michelson, and X. Wu, Dynamics of antimembranes in the maximally supersymmetric eleven-dimensional pp-wave, J. High Energy Phys. (2006), 028, 37 pp.

[11] V. Pravda, A. Pravdova, A. Coley, and R. Milson, All spacetimes with vanishing curvature invariants, Classical Quantum Gravity 19 (2002), 6213-6236.

[12] Th. Leistner, Screen bundles of Lorentzian manifolds and some generalizations of pp-waves, J. Geom. Phys. 56 (2006), $2117-2134$. 
[13] M. Brozos-Vázquez, E. Garcia-Rio, P. Gilkey, S. Nikčević, R. Vázquez-Lorenzo, The Geometry of Walker Manifolds. Synthesis Lectures on Mathematics and Statistics, Vol. 5, Morgan and Claypool Publ, 2009.

[14] Y. Matsushita, Walker 4-manifolds with proper almost complex structures, J. Geom. Phys. 55 (2005), 385-398.

[15] M. Brozos-Vázquez, E. Garcia-Rio, and R. Vázquez-Lorenzo, Conformally Osserman four-dimensional manifolds whose conformal Jacobi operators have complex eigenvalues, Proc. R. Soc. A. 462 (2006), 1425-1441.

[16] P. Gilkey, The geometry of curvature homogeneous pseudo-Riemannian manifolds. ICP Advanced Texts in Mathematics, 2. Imperial College Press, London, 2007.

[17] M. Nadjafikhah, M. Jafari, Some general new Einstein Walker manifolds, arXiv:1206.3730v1 [math.DG] 17 Jun 2012.

[18] S.V. Meleshko, Methods for Constructing Exact Solutions of Partial Differential Equations, Springer Science+Business Media, Inc, NewYork, 2005.

[19] A.M. Grundland, L. Lalague, Invariant and partially-invariant solutions of the equations describing a non-stationary and isentropic flow for an ideal and compressible fluid in $(3+1)$ dimensions, J. Phys. A: Math. Gen. 29 (1996) 1723-1739.

[20] A.M. Grundland, P. Tempesta, P. Winternitz, Weak transversality and partially invariant solutions, J. Math. Phys. 44(6) (2003) 2704-2722. 